\title{
Cost-oriented Context and Content Data Pair Delivery in Smart PIN
}

\author{
Seung-Bum Lee ${ }^{1,2}$, Gabriel-Miro Muntean ${ }^{1}$, and Alan F. Smeaton ${ }^{2}$ \\ ${ }^{1}$ Performance Engineering Laboratory (PEL) \\ ${ }^{2}$ Centre for Digital Video Processing (CDVP) \\ ${ }^{1,2}$ Dublin City University, Glasnevin Dublin 9, Ireland \\ \{sblee, munteang\}@eeng.dcu.ie \\ alan.smeaton@computing.dcu.ie
}

\begin{abstract}
The latest evolutions in wireless technologies and advances in mobile devices enabled user devices to have access and acquire large amounts of data. Metadata and algorithms are required for users to be able to handle such large sets of data. For this purpose, this paper introduces Smart PIN - a novel performance and cost-oriented, context-aware personal information network. Smart PIN architecture includes network, service and management components. At the service components, there should be consideration for service discovery, service composition, data replication management and data pair transfer. This paper also proposes a novel scheme for efficient delivery of context and content data based on pull and push scheme controlling logical and physical cost function.
\end{abstract}

Keywords: Context-awareness, Data replication, Cost-effectiveness, Wireless PAN.

\section{Introduction}

The latest wireless technologies are offering more bandwidth, support for higher mobility and lower power consumption while providing various connectivity. Services are also becoming personalised, more diverse and automatic across diverse types of devices such as consumer electronic products, personal computers and mobile devices. However, users still need to struggle to access the data they want since it is distributed across various the devices users may have. The existing solutions such as network file systems can only provide restricted access to the wanted content and users need to manipulate specific devices offering the required service.

The difficulties to make service access simple are mostly due to the huge amounts of data acquired and stored in distributed devices. Furthermore, it is not difficult to realise that services are limited to user devices and these can provide meaningful information to users if the point of view is moved to the user. In this context, Wireless Personal Area Networks (WPAN or Wireless PAN) are human centred networks connecting devices in a spontaneous architecture within a short-rage ("personal" or "body" space) [1] and they provide a good infrastructure for integrating services. For WPAN, there are some technologies proposed or already implemented such as Bluetooth [2] and IEEE 802.15 [3]. Since users can not manage large amounts of data manually, they require an intelligent solution to solve this problem. Metatdata, data about data, helps systems to mitigate user intervention. Furthermore, context information can be used as metadata in multimedia services [4] and users can access contents through the context instead of exact file names [5].

This paper presents a solution to this problem by proposing, Smart PIN (a Performance and costoriented context-aware personal Information Network). Smart PIN is a novel solution that enables efficient user access to information located on remote devices. Fig. 1 presents an overview of the proposed Smart PIN. It includes a number of agents that communicate using various network technologies 


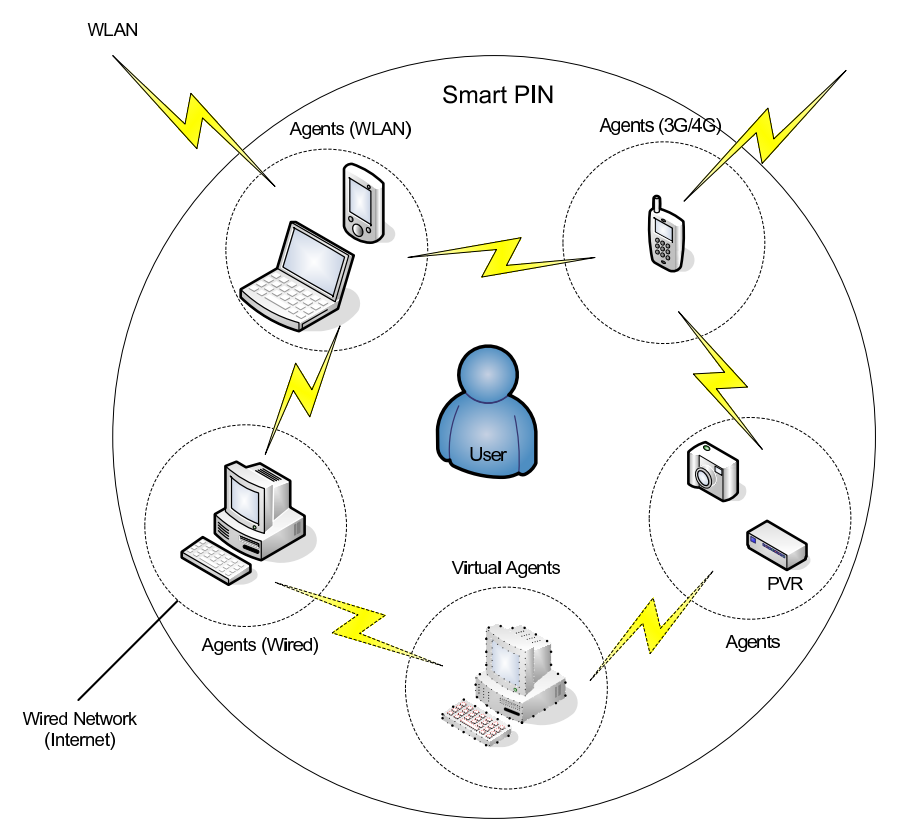

Figure 1: Topology of Smart PIN

and provide support for information exchange. Each agent may have network connectivity with entities outside the Smart PIN space. Especially, this paper proposes novel performance and cost-oriented context and content data pair delivery in Smart PIN.

This paper is structured as follows. The following section describes related work which presents similar approaches to Smart PIN. Section 3 introduces the proposed Smart PIN with focus on efficient delivery of context and content data pairs. Preliminary simulation tests, results analysis and conclusions follow at the end of the paper.

\section{Related Works}

In relation to the network infrastructure, there are a few approaches based on WPAN. Personal networks (PNs) [6], for example, refer to the extension of the personal area network to enable co-operation with remote devices and services. Furthermore, Fednet was proposed as an ad hoc federation of independent PNs. A federation in Fednet could be considered as a trust-based access control scheme of shared resources. Also with this concept, the My personal Adaptive Global NET (MAGNET) project [7] basically focuses on network layer issues.

Ambient network (AN) [8], is the another approach based on WPAN, and could be considered as a control plane framework focusing on an automatic roaming agreement and composition of networks. For these features, AN utilises an AN interface (ANI) and builds an AN Control Space (ACS) using a Generic AN Signalling protocol (GANS). In AN, there are three Functional Areas (FA): Composition FA, Mobility FA and QoS FA in order to support these mentioned features. AN is also trying to focus on self organisation from the view of the service provider.

As none of above works introduce context and content distribution performance and cost, Smart PIN extends these concepts beyond MAGNET and AN to application layers by offering a performance and cost-related solution to the problem for efficient delivery of data. 

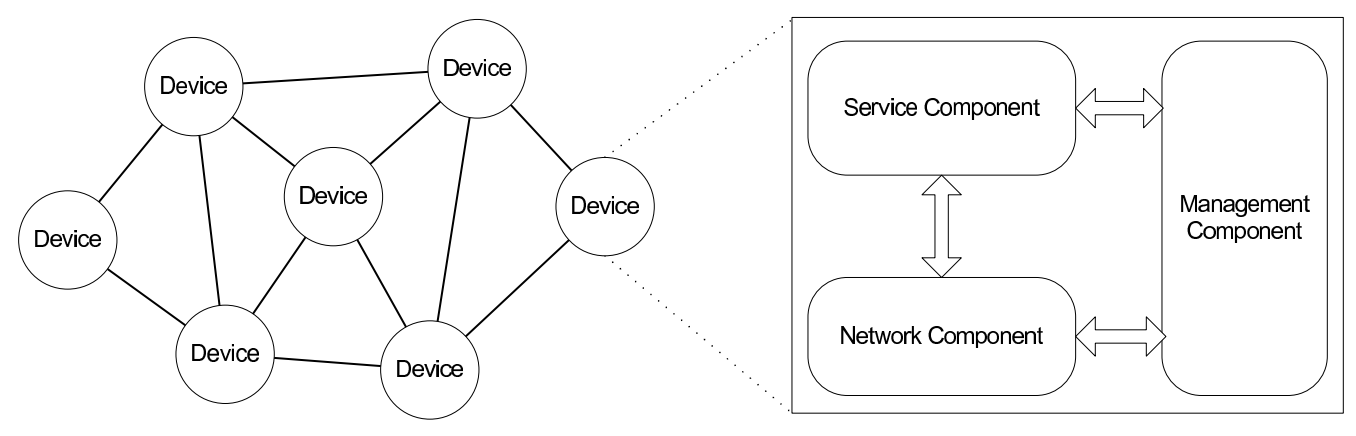

Figure 2: System Architecture Diagram

\section{Smart PIN - Performance and Cost-Oriented Context-Aware Personal Information Network}

The proposed Smart PIN, a performance and cost-oriented context-aware personal information network, is a novel solution that enables efficient user access to information located on remote devices. Smart PIN handles data pairs that consist of informational context and content, as similarly described by the digital items in MPEG-21 [9]. As a context-aware system, Smart PIN focuses on removing any unnecessary user interaction, while providing performance and cost benefits. In Smart PIN, it is assumed that same categories of informational context and content exist in distributed sources. For example, a user could have emails which are stored on a desktop at their house, on a laptop at work and on webmail as part of a blog service. For user convenience the integration of these services is very desirable.

\subsection{System Architecture}

As Fig. 2 schematically presents, Smart PIN is composed of a number of interconnected distributed devices. Each has three major components: Network, Service and Management.

The network component covers vertical homogeneous interactions between physical and link network layers for each technology. It is also concerned with heterogeneous network interaction through network layer bridges. Furthermore, it looks at automatic configuration and self-management in heterogeneous networks. Smart PIN involves a virtual agent in order to invite external services (e.g. Blogging) into the system. Support for personal privacy and security is also provided. Autoconfiguration and Selfmanagement of Personal Area Networks (ASPAN) [10] is one approach for automation of network layers that can be employed. However this needs to be considered in relation to the other components.

The service component focuses on service discovery, service integration, search and delivery for context and content, and access control protocols. The services need to be integrated and this service integration is responsible for searching distributed sources for digital items, managing integration of services and responding to client requests for integrated services. Applications search and transport the context and contents through usual file transport protocols for the integrated services. For overall services, various access controls could be applied for maintaining user privacy.

The goal of the management component is to support cost effectiveness in various aspects of the system that operate in an heterogeneous network environment. Cross-layer approaches such as [11] could be considered here. The context and status of networks and services could be gathered here and could be used for QoS, power efficiency or service differentiation.

Smart PIN should work not only with devices capable of heterogeneous network connectivity, but also with those devices supporting a single wireless network technology. Similar to the usual PAN-based solutions, it is envisaged that multiple Smart PINs could co-operate with each other for exchanging not only context and content, but also for interacting sensors and other services. 


\subsection{Service Scenarios}

The scenarios examined here are related to service component assuming that other network conditions are provided. In this situation, accessing context and content data pair could be possible after services are found and integrated; subsequently available data will be composed. With this sequence of events, possible service scenarios are as follows:

- Service status change: This category of scenarios is for handling movement of devices, or change of services. For example, a device joins an integrated service assuming it is not already involved in an integrated service or is integrated within another service. Another scenario involves a device leaving an integrated service. Furthermore, several devices could be connected via different networks. If there is extra available connectivity between these networks, these devices could join an integrated service. These issues will be discussed in detail in the service discovery and several service composition management.

- Data pair change: This category of scenarios is related to changes of context and content data pairs. In an integrated service, new data can be produced via direct generation or transfer from other devices. The deletion of existing data is also one scenario which affects the data pairs. Not only for creation and deletion of data in one device, but also available data expansion induced from integrating services is a significant scenario resulting from service status change. More simply, one or more devices which have specific amount of data pairs, joins the services. Then, the total available data pairs can be defined as a superset of data among the connected devices. This issue will be discussed in detail in the data pair set-management and data pair transfer section.

\subsubsection{Service Discovery and Service Composition Management}

For handling service state change, the services in mobile devices needed to be found first. There are several proposed protocols like SDP for Bluetooth [2] and SSDP for UPnP [12] which focus on service discovery. Existing service discovery mechanisms are usually adopting a broadcast message such as WiFi beacon, advertisement and solicitation IP messages, etc. On the other hand, the network component in charge of the remote device requires a unicast based service discovery mechanism since the connectivity used between those is not proper for broadcasting. With this context, the selection of a proper protocol type needs to be considered next with characteristics of the network components layer.

When services are discovered, the device should be involved in service composition to provide an integrated service. Furthermore, a device can leave the network at any time and lose the connection and the remaining devices should update their status. Briefly, service composition is concerned with building up an integrated service with services through service discovery. With this context, this issue has quite a deep relationship with network composition caused by device inclusion/exclusion and network merge/separation scenarios. A good example for network composition is introduced in [13] and ASPAN [10] presents dynamic and automated network layers management on an Ambient Network. With these schemes, the assumption that all devices have the same service enables management of network and service composition at the same time. However, structural design issues should be considered carefully based on a cross-layer approach.

\subsubsection{Data Pair Set-Management and Transfer}

The available data pairs in Smart PIN could be defined as a set of total data pairs in the integrated services. During data pair transmission, duplication can occur. Furthermore, joining of a device or merging of two or more networks requires an efficient management scheme for keeping updates of available data pairs in the system.

In the view of management of distributed data, there could be a centralised approach such as OmniStore [5] or a distributed approach like Bayou [14]. There is also a hybrid model such as Roam [15]. During service state changes, devices maintain a data set model which is very similar to the WARD 


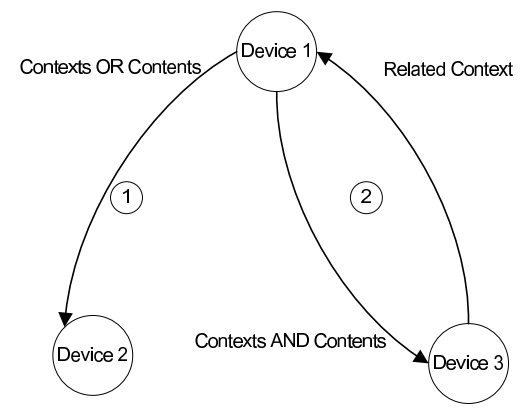

Figure 3: Types of data delivery in Smart PIN

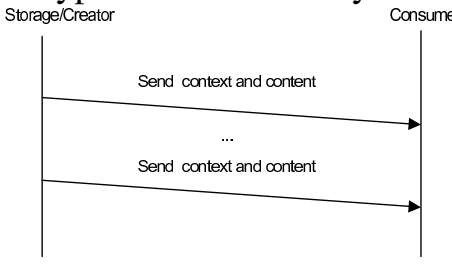

Figure 4: File push ladder diagram

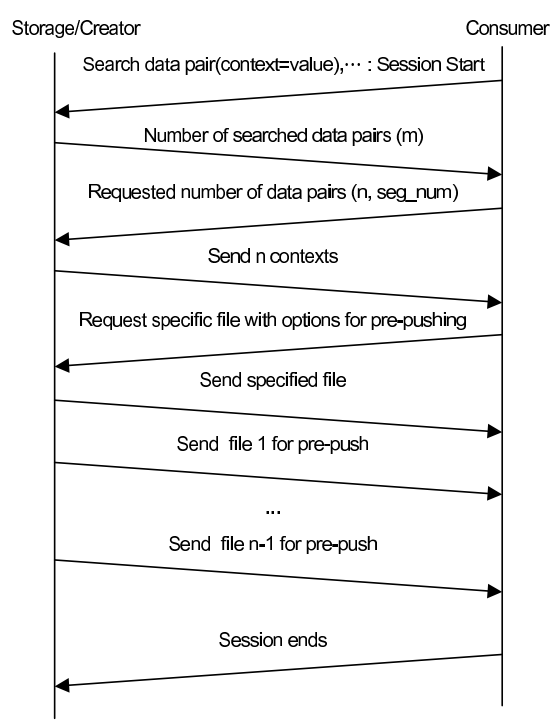

Figure 5: File pull ladder diagram

model [16]. However, devices in Smart PIN have different characteristics and the model needs to consider those characteristics for improving performance and efficiency. For example, if a powerful server is available, it is chosen as the master for the integrated service, consequently the mobile devices will benefit if most transmissions occur via the server. Alternatively, a dynamic approach utilizing centralised approach or hybrid peer-to-peer can be used.

For the data pair change which is mentioned above, data pair transmission acts as one main role of the scenarios. When a data pair is generated, contexts are needed to be shared to make that pair a member data of the integrated service. As data is pushed or pulled, contents are also transferred to some other device in near proximity. Similarly, this operation is employed for data browsing. During data deletion, actual content delivery is not required. Only context is needed to be transferred to update status of data replication. If there is no other device having this content, then that context and content data pair is really deleted. When a new member device joins the network, there could be many new data pairs which are not included in the integrated service. However, there are the only differences in the amount of data which could be processed with either push or pull mechanism. Most schemes for data replication for mobile devices $[5,15]$ focus on the delivery and replication scheme for files but do not consider the cost of delivery. The detailed scheme for data pair transfer in Smart PIN will be discussed in section 3.3.

\subsection{Efficient Context and Content Delivery}

Fig. 3 shows the main steps in distributing these data pairs. Usually, a device pulls data identified by a specific context (2) in Fig. 3 and Fig. 4). If the device user has a certain level of interest in a piece of content, both context and content are transferred, otherwise only the context is delivered. Alternatively, context or context-content data could be pushed to specific devices (1) in Fig. 3 and Fig. 5), too.

If there is a device which has large storage space, a backup scheme could be considered as a pushbased replication application. Most systems usually transfer context first, however the delivery of the actual content could also be transferred from a single device or multiple devices. If there is no space for new generated context and content, deletion of files which are already replicated and the least used can be automatically performed. In push-based data replication, Smart PIN uses the push triggering memory ratio (PTMR). The acquirable free space is defined as the free space plus the space for replicated files which can be deleted if required. The device checks whether the maximum acquirable free space ratio (i.e. ratio between acquirable free space and the total existing memory) exceeds PTMR periodically. If it does, the device starts pushing of files until it reaches an enough number of pushed files. PTMR is defined as in the following equation (1) 


$$
P T M R=M F S R+(1-M F S R) \times C(t)
$$

In (1), MFSR stands for the minimum free space ratio which represents the minimum memory space to be saved for storing new context-content data pairs. With (1), PTMR is greater than or equal to MFSR. The $C(t)$ is a performance-cost function defined as in equation (2):

$$
\begin{gathered}
C(t)=\max _{t}\left(c_{i}(t)\right) \\
c_{i}(t)=\frac{\alpha L_{i}(t)+\beta P_{i}(t)}{\alpha+\beta}, \text { with } \alpha+\beta=1 \\
L_{i}(t)=\frac{l_{i}(t)}{\max _{i}\left(l_{i}(t)\right)}, P_{i}(t)=\frac{p_{i}(t)}{\max _{i}\left(p_{i}(t)\right)}
\end{gathered}
$$

$l_{i}(t)$ is the logical cost function for communication that includes access cost (e.g. free, fixed rate or packet based charges) or any other user definable communication-related cost function. $p_{i}(t)$ is the physical resource-based cost function and involves energy consumption and bandwidth. After normalisation using equation (4), $l_{i}(t)$ and $p_{i}(t)$ can be presented as $L_{i}(t)$ and $P_{i}(t)$. These functions have values from 0 to 1 and refer to the performance-cost function in relation to $\mathrm{n}$ network communication technologies.

The pull scheme is involved in usual data search and browsing as presented in the Fig. 5 ladder diagram. The number of data pairs will be higher than one since it is accessed with specific context related information not with an exact file name. However, transferring all searched data pairs to the user device is not efficient. Furthermore, controlling the number of contexts and contents to transfer is a key issue for the pull scheme. In Fig. 5, when the consumer get the number of searched data pairs (m), he will determine the maximum number of possible pre-push data (n) and request a segment having prices of data with $\mathrm{n}$ smaller than $\mathrm{m}$. The number of files actually pulled $N_{p p}$ is determined by the storage device with equation (5).

$$
N_{p p}=\lfloor n \times C(t)\rfloor
$$

\section{Simulation Test and Results Analysis}

\subsection{Simulation Test}

The proposed scheme for delivery of content-context data pairs is evaluated via network simulation using Network Simulator 2 (NS-2) [17], a discrete event simulator which provides substantial support for the simulation of data delivery using various protocols in wired and wireless networks. A IEEE 802.11b WLAN was simulated with maximum rate of $11 \mathrm{Mbps}$ at $2.4 \mathrm{GHz}$ band using DSDV for ad-hoc routing.

Content and context data acquisition was randomly generated using an exponential distribution with a mean of 10 seconds. For data context, the assumed size was uniformly distributed between 1 and 10 Kbytes. For the generation of content, the size was uniformly distributed between 400 Kbytes to 3 Mbytes. Data pairs were provided at specific times to the simulator. For data initially stored on the device the time was set to 0 . Simulation topology includes one device for storage and two devices for providing data pairs. The parameters used in simulation were 40 Mbytes for memory size, 0.2 for MFSR, 0.6 for desired free memory ratio (DFSR) and 0.5 secs for buffer check period.

\subsection{Results Analysis}

Fig. 6 presents the total memory usage variation plotted against time. All situations have the same graph since it adopts the same deletion scheme based on a constant threshold which is similar to OmniStore. Since the deletion threshold, MFSR (Minimum Free Space Ratio) is 0.2, the maximum occupied memory can reach 32 Mbytes. If the memory used becomes greater than 32 Mbytes, the deletion process acquires 


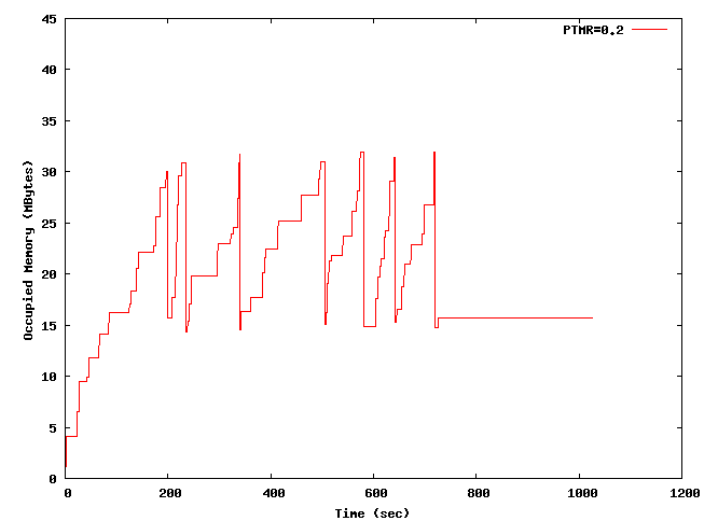

Figure 6: Memory used for storing (context-content) data pairs

\begin{tabular}{|c|c|c|c|c|c|}
\hline \multirow{2}{*}{ Scheme/PTMR } & \multicolumn{2}{|c|}{ Queued files(MB) } & \multicolumn{2}{c|}{ Pushed files(MB) } & \multirow{2}{*}{ Loss } \\
\cline { 2 - 5 } & Avg. & Std. Dev. & Avg. & Std. Dev. & \\
\hline PTMR $=1.0$ & 0 & 0.00 & 19.20 & 5.60 & 0.00 \\
\hline PTMR $=0.8$ & 7.24 & 0.83 & 11.96 & 5.44 & 0.00 \\
\hline PTMR $=0.6$ & 14.71 & 2.07 & 4.49 & 5.06 & 0.00 \\
\hline PTMR $=0.4$ & 18.30 & 4.34 & 0.90 & 2.09 & 0.11 \\
\hline PTMR $=0.2$ & 19.20 & 5.60 & 0.00 & 0.00 & 0.25 \\
\hline \hline Periodic Push & 0 & 0.00 & 19.20 & 5.60 & 0.00 \\
\hline No push & 19.20 & 5.60 & 0.00 & 0.00 & 0.25 \\
\hline
\end{tabular}

Table 1: Simulation test results

free space up to DFSR. For this case, free memory needs to be 24 Mbytes. Furthermore, the occupied memory reduces up to 16 Mbytes. If there are no pushed files, then the files which are the least used are chosen for deletion.

Table 1 presents a statistical analysis of memory usages with variation of PTMR and other approaches such as periodic push without cost evaluation and no push. If PTMR has a high value such as 0.8 for low cost, the size occupied by queued files which are not pushed and stored in devices are decreased and most are replicated to other devices. In contradiction, if PTMR has low values such as 0.2 for high cost, the queued files are increased. Furthermore, pushed files are decreased under this condition. Because of the deletion scheme, there is a high loss as those files are not replicated. As the results show, the memory size of queued files and pushed files varies according to cost function. Since pushed files represent amount of data which is transferred as push data, cost function can control replication overhead traffic.

\section{Conclusion}

This paper proposes Smart PIN, a novel performance and cost-oriented context and content data pair delivery mechanism. It also presents the Smart PIN system architecture with its major components: network, service and management components. For the service component, different scenarios were presented as service status and data pair change. Furthermore, potential algorithms were discussed.

Context and content data delivery in the service component were evaluated through network simulation. Between push and pull schemes, a cost-dependent threshold distinguishes between the delivery costs and provides differentiated processing of push-based delivery of data for file replication. Work on how to realise a cost function for the proposed schemes is in progress. For the service component level such as service discovery, service composition, data replication management, context dissemination and access control, different solutions will also be evaluated. With those evaluations, network and management components will be involved to increase the performance and cost-effectiveness of the solution, 
especially with regard to transporting multimedia data. Prototyping is also envisaged as a long term objective.

\section{References}

[1] T. B. Zahariadis, "Evolution of the wireless pan and lan standards," Computer Standards and Interfaces, vol. 26, no. 3, pp. 175-185, May 2004.

[2] Bluetooth SIG, http://www.bluetooth.com/bluetooth/.

[3] "IEEE 802.15 working group for wireless personal area network," http://www.ieee802.org/15/.

[4] M. Davis and et al, "MMM2: mobile media metadata for media sharing," Conference on Human Factors in Computing Systems, pp. 1335 - 1338, 2005.

[5] A. Karypidis and S. Lalis, "OmniStore: A system for ubiquitous personal storage management," Pervasive and Mobile Computing Journal, 2007, to appear (accepted for publication), Elsevier.

[6] I. G. Niemegeers and S. M. H. D. Groot, "Fednets: Context-aware ad-hoc network federations," International Journal of Wireless Personal Communications, vol. 33, no. 3-4, pp. 305-318, Jun. 2005 .

[7] “MAGNET,” http://www.telecom.ntua.gr/magnet/index.html.

[8] C. Kappler, P. Mendes, C. Prehofer, P. Pöyhönen, and D. Zhou, "A framework for self-organised network composition," Lecture Notes in Computer Science, vol. 3457, pp. 139-151, Jul. 2005.

[9] I. Burnett, R. V. Walle, K. Hill, J. Bormans, and F. Pereira, "MPEG-21: Goals and achievements," IEEE Multimedia, vol. 10, no. 4, pp. 60-70, Oct.-Nov. 2003.

[10] R. Campos and M. Ricardo, "Dynamic and automatic connection of personal area networks to the global internet," The International Wireless Communications and Mobile Computing Conference, pp. $581-586,2006$.

[11] M. Conti, G. Maselli, G. Turi, and S. Giordano, "Cross-layering in mobile ad hoc network design," IEEE Computer Magazine, vol. 37, no. 2, pp. 48-51, Feb 2004.

[12] "Understanding Universal Plug and Play," http://www.upnp.org/download/UPNP_UnderstandingUPNP.doc.

[13] J. Rey, B. Mathieu, D. Lozano, S. Herborn, K. Ahmed, S. Schmid, S. Goebbels, F. Hartung, and M. Kampmann, "Media aware overlay routing in ambient networks," Personal, Indoor and Mobile Radio Communications, pp. 952- 957, Sep. 2005.

[14] A. J. Demers, K. Pertersen, M. J. Spreitzer, D. B. Terry, M. M. Theimer, and B. B. Welch, "The bayou architecture: support for data sharing among mobile users," IEEE Workshop on Mobile Computing Systems \& Applications, pp. 2-7, 1994.

[15] D. Ratner, P. Reiher, and G. J. Popek, "Roam: a scalable replication system for mobility," Mobile Network and Applications, vol. 9, no. 5, pp. 537-544, Apr 2004.

[16] D. Ratner, P. Reiher, G. Popek, and G. Kuenning, "Replication requirements in mobile environments," Mobile Networks and Applications, vol. 6, no. 6, pp. 525-533, Nov 2001.

[17] “Network Simulator 2,” http://nsnam.isi.edu/nsnam/index.php/Main_Page. 